\title{
CONSULADOSY SERVICIOS SECRETOS ALIADOS EN MELILLA DURANTE LA SEGUNDA GUERRA MUNDIAL
}

\author{
Santiago Domínguez Llosá \\ Círculo Naval Español \\ saludollo@gmail.com \\ "En memoria de D. Manuel Cuenca Toro"
}

Resumen: El objeto de este trabajo es hacer una aproximación a la presencia en Melilla de representaciones diplomáticas de los aliados en la II Guerra Mundial, así como la actuación en la zona de sus servicios secretos, el Intelligence Service inglés y la Office Special Services norteamericana.

Palabras Clave: Melilla, II Guerra Mundial; consulados; servicios secretos.

Abstract: The topic of this essay is an aproximation to the presence in Melilla for alied diplomatic representations in the II World War, as well as the intervention in the área of their secret services, english Intellicence Service and american Office Special Services.

Keywords: Melilla, Second World War, consulates, secret services.

\section{Introducción}

Como ocurrió en los dos conflictos mundiales, los países neutrales se convirtieron en centros de información privilegiados para los contendientes. Y la ciudad de Melilla, durante la Segunda guerra a Mundial, dada su cercanía a la Zona francesa, y ser el puerto desde donde salían barcos cargados de mineral de hierro de las minas del Rif, especialmente para Inglaterra y sus aliados, no fue una excepción. Además, tras la invasión aliada del norte de África en noviembre de 1942, Melilla se convirtió en la principal "antena" del Eje para obtener información sobre los movimientos aliados. 


\section{I.- CONSULADOS Y VICECONSULADOS ALIADOS EN MELILLA.}

\section{Viceconsulado Británico.}

Ya en 1919 se trató por parte del Gobierno inglés la instalación en Melilla de un consulado o representación diplomática ${ }^{1}$, pero no es hasta bien entrada la II Guerra Mundial, cuando se plantea seriamente su instalación. [1]

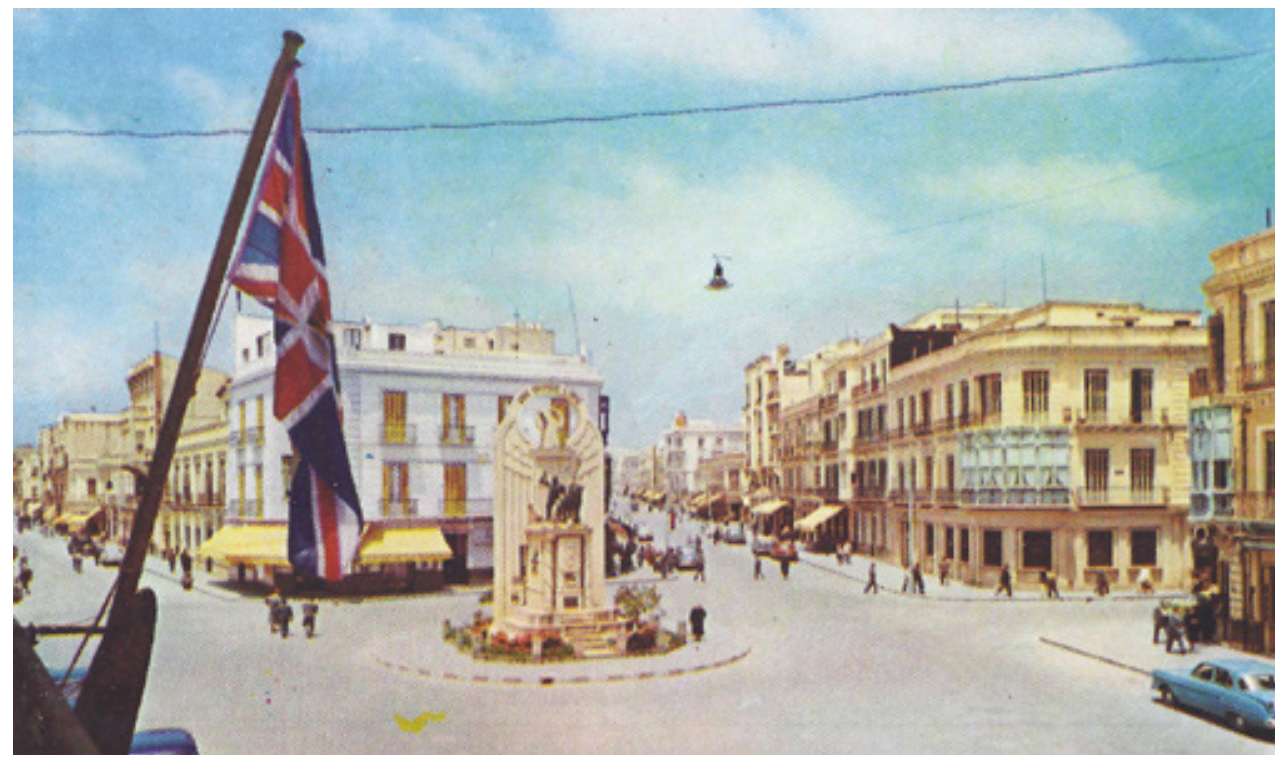

Fig. 1. Vista de la Avenida desde el viceconsulado inglés en Melilla, con la Union fack en primer término. La fotografía está tomada en los años 50,justo antes del cierre de sus oficinas.

El motivo para la implantación de esta oficina consular fue, en principio, para facilitar la obtención de los preceptivos "Navicert" a los comerciantes melillenses, que hasta ese momento se veían obligados a solicitarlos al lejano consulado británico de Tetuán. Sin embargo, para los servicios secretos españoles el motivo principal de su establecimiento sería la de facilitar el trabajo de los miembros del "Intelligence Service" de la zona ${ }^{3}$.

El 25 de febrero de 1941, se recibe en el Ministerio de Asuntos Exteriores español la propuesta de la Embajada Británica, proponiendo a Richard Croker como vicecónsul de Gran Bretaña en Melilla. A partir de ese día, se empiezan a intercambiar toda una serie de oficios entre los distintos servicios del Protectorado solicitando antecedentes e 
información sobre él ${ }^{4}$. El 18 de marzo de 1941, la Sección de Vigilancia y Seguridad de la Delegación de Asuntos Indígenas de Tánger contesta que han resultado infructuosas las gestiones encomendadas para obtener información, "al ser desconocido en esta localidad y carecer de antecedentes en el Archivo de la Comisaría central de Policía".

Tiempo más tarde, el 5 de junio de 1941, el gobierno español concede una autorización provisional para que el citado ejerza el cargo señalado por el gobierno británico, haciéndoselo saber a las autoridades competentes para que "se admita al interesado en el ejercicio de sus funciones"5; pocos días después, el 11 de junio llega a Melilla el nuevo vicecónsul, alojándose en el Hotel España.

La primera oficina del consulado británico se instaló en el número 4 de la calle Alfonso XII, en el Mantelete, en un edificio propiedad de J. Salama. Posteriormente, el vicecónsul pasó a residir a la calle Polavieja, en una casa que le concedió la fiscalía local de la Vivienda, trasladándose la oficina consular a la Plaza de los Héroes de España. [2]

Sobre el perfil y semblante del nuevo cónsul, la ficha elaborada por el servicio de información de la Alta Comisaría lo describe como "de 40 años, natural de Canarias, dice ser casado y de profesión Diplomático ${ }^{\prime}$. Realizándose observaciones al respecto a su vida privada, era señalado como "un tanto vicioso, frecuenta los prostíbulos, pasando muchas noches con mujeres de mala nota $"$. En otro informe, con fecha 16 de junio de

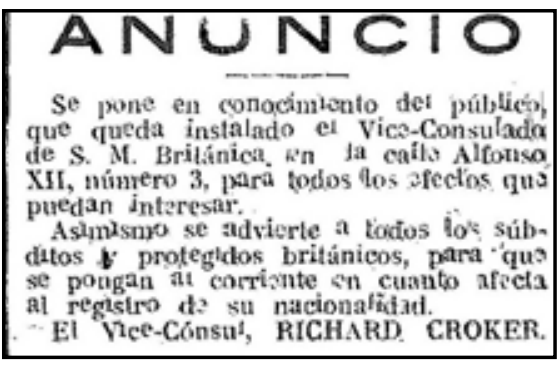

Fig. 2. Anuncio publicado en El Telegrama del Rif en el que se comunica la apertura del viceconsulado inglés en Melilla.

1 The National Archives (NA), Kew. Consular Services: Proposed establishment of British Consulate at Melilla. 1919. FO 608/175/14.

2 Navicert: en el Derecho Maritimo Internacional se conoce con dicho nombre el salvoconducto que, en tiempo de guerra, concede una nación a los barcos de países neutrales para que puedan realizar el comercio maritimo por no transportar mercancias perjudiciales para el beligerante que lo expide o con origen o destino a países enemigos. Navegar sin el correspondiente navicert exponía a los barcos a ser detenidos en alta mar por la Royal Navy y dirigidos a puerto.

3 Archivo General de la Administración (AGA), 81/4190: Exp. personal de Richard Croker.

$4 \quad$ AGA. 81/5896: Expediente Personal de Ricardo Croker.

5 AGA. 81/4190: Expediente Personal de Richard Croker.

6 AGA. 81/4190. Expediente Personal de Richard Croker.

7 AGA. 81/4190. Expediente Personal de Richard Croker. 
1942, va más lejos en la calificación de sus costumbres privadas "se dedica a recibir en su casa a prostitutas, que sacaba de las casas de lenocinio, despidiéndolas a las cuatro de la madrugada, las cuales, a pesar de ser pagadas espléndidamente, no querían volver, debido a los muchos vicios del personaje que nos ocupa". En ese mismo informe, también se hace una insinuación muy directa a su posible bisexualidad, indicando que "se sabe que tiene amistad muy íntima con el súbdito griego GEORGE E. AMANATIDES, vigilado por estos servicios de Policía; estando dicho súbdito griego considerado como invertido y pasando muchos ratos en reunión íntima con el inglés, completamente solos y ataviados con albornoces $8 "$.

Varios meses después de su llegada, el 10 de febrero de 1942, venía a Melilla Natividad Pulgar Gómez, una española de 27 años de edad, a la cual intentó hacer pasar por su esposa, aunque parece ser que en realidad era su amante. Semanas después de su llegada, tuvo la desdicha de caer enferma, falleciendo en Melilla el 12 de marzo de ese mismo año. Richard Croker se hizo cargo de todos los gastos, tanto de hospitalización, como del entierro, pero se encargó de que no apareciese su nombre en ninguno de los documentos relacionados con Natividad ${ }^{9}$.

De cara a su vida pública, fue reconocido como persona afable, culto y demócrata. En su ficha personal leemos que "conoce perfectamente el español y es muy amante de las costumbres españolas, especialmente de las andaluzas; es hombre simpático y de franca cordialidad, aficionado a la juerga, sobre todo si esta es de carácter español”. [3]

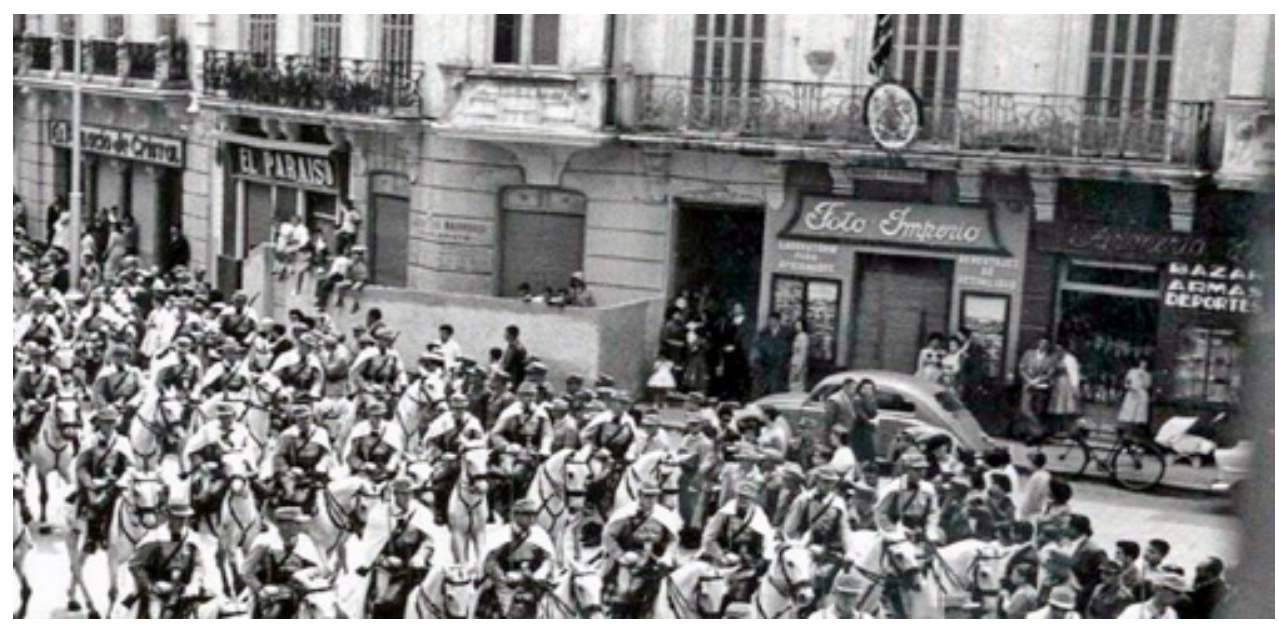

Fig. 3. Desfile de fuerzas de Regulares de Caballería. Sobre el comercio Foto Imperio, se aprecia el escudo y la bandera inglesa. 
Con respecto a sus actividades profesionales, fue un estricto servidor de los intereses ingleses, teniendo continuos enfrentamientos con autoridades, comerciantes y consignatarios de buques. A su llegada, y ya en la primera reunión que tuvo con las autoridades exigió que nadie se acercase, y ni mucho menos se fotografiase, a los barcos ingleses que venían a cargar mineral de hierro; debido a haber ocurrido un grave incidente con uno de los miembros de los servicios secretos alemanes ${ }^{10}$ que se dedicaba a ello en el puerto melillense, que acabo apaleado por parte de la tripulación de uno de los barcos. Posteriormente, protagonizó otros incidentes con las autoridades al realizar viajes por la zona próxima a Melilla con fines poco claros y sospechosos para los Servicios de Inteligencia españoles ya que en ellos se aproximaba mucho a zonas consideradas de interés para la defensa y donde se estaban construyendo obras de fortificación. [4]

Algunos de estos incidentes fueron verdaderamente tan ridículos como exagerados. Por ejemplo, en julio de 1942, cuando se presentó en Cala Charranes para pasar una jornada típica de verano en la playa. Esto motivó inmediatamente toda una serie de mensajes cruzados entre las autoridades militares de Melilla, la Alta Comisaría y el Ministerio de Asuntos Exteriores, sobre si se le debía permitir o no el acceso del cónsul a dicha playa. Tras un mes de consultas, se decidió que se le podía permitir acceder a la misma al no ser considerada lugar de interés para la defensa nacional.

Con respecto a su relación con los comerciantes, realizó una "lista negra" de todos aquellos que tenían

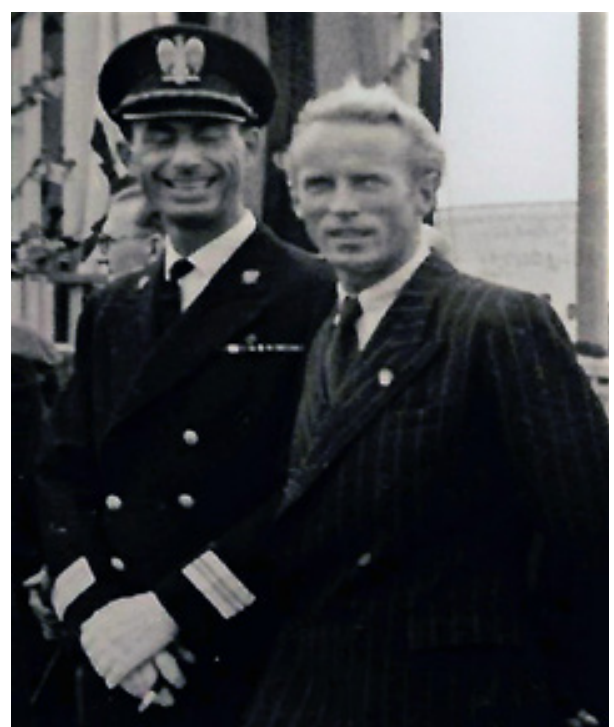

Fig.4. El oficial de la Kriegsmarine Fritz Lackver, miembro de los servicios secretos alemanes en Melilla, donde era conocido como "el rubio de la moto" $y$ que protagonizo varios incidentes con súbditos ingleses. funto a él, de uniforme, el comandante de la marina italiana Giuseppe Mondini, jefe de la misión militar italiana en Melilla.

\footnotetext{
8 AGA.81/4190. Expediente Personal de Richard Croker, Informe reservado de la jefatura Local de Vigilancia y Seguridad, dirigido al Interventor Regional de Nador. Archivo del Cementerio de la Purísima Concepción de Melilla, Libro de Enterramientos (1942), Asiento $n^{\circ} 276$.

10 Se trataba de Fritz Lackvner, conocido en la ciudad como "El rubio de la moto", oficial de la Kriugsmarine encargado del control de los barcos aliados que entraban en Melilla.
} 
alguna vinculación con alemanes o italianos, como por ejemplo con la firma Tortosa y López Ávalos (representantes de Telefunken). Al gerente del hotel donde se hospedaba, lo amenazó, medio en broma medio en serio, con incluirlo en la citada lista si admitía a huéspedes de dichas nacionalidades. Lo mismo ocurrió a la hora de conceder los preciados permisos "Navicert" debido a su celo, teniendo continuos enfrentamientos con los agentes de transportes, a los que sistemáticamente negaba dichos permisos si no iban perfectamente documentados. Este carácter "impulsivo y despótico en cuanto se trata de asuntos oficiales y su intransigencia ha creado en Melilla un ambiente que hace a todos ver que Inglaterra es la dueña del mundo y que nada se hará sin su consentimiento" ${ }^{11}$ ”.

Dicha actitud le granjeó la enemistad de numerosas personas en la ciudad. Por lo que no fue extraño, que, debido a la polémica explosión de la valija inglesa en Tánger, en la mañana del día 12 de febrero de 1942, la fachada del Consulado inglés en Melilla apareciera cubierta de pintadas que decían: "GIBRALTAR"; "EL HAMBRE DE ESPAÑA SE LE DEBE A INGLATERRA"; "OJO, AQUÍ HAY VALIJA INGLESA"; "ESPAÑA, GIBRALTAR", "SINGAPOORE, JAPÓN"12 ", cuya autoría no pudo esclarecerse.

Otras actividades del viceconsulado, además de las relacionadas directamente con el comercio, fue el reparto de propaganda pro-aliada; labor siempre dificultada por los servicios españoles. A la hora de repartir propaganda, se usaba cualquier método: desde importar y repartir paquetes de té con la bandera inglesa, a la distribución de revistas y publicaciones en español que ensalzaban los avances aliados. También mediante el reparto de ropa, especialmente jerséis con la "Union Jack" (Bandera del Reino Unido), entre los trabajadores del puerto y llevada

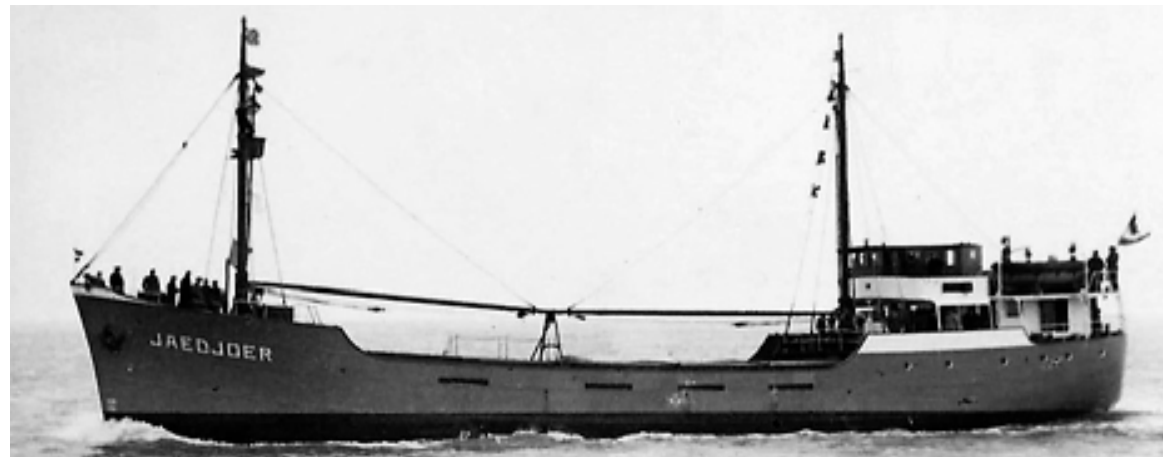

Fig. 5 Mercante alemán AAEDFOER, que con el nombre falso de LOLA entró en el puerto de Melilla en diciembre de 1941. Su estancia fue objeto de vigilancia y de la planificación de su destrucción por parte de los servicios especiales ingleses. 
a cabo por las tripulaciones de los barcos ingleses que recalaban en el mismo. [5]

En enero de 1942, se produce el llamado "Incidente LOLA/ JAEDJOER ${ }^{13}$ " protagonizado por el barco de dicho nombre al servicio de Alemania, pero con bandera española. Refugiado en el puerto de Melilla cuando se dirigía a Libia para aprovisionar al "Afrika Korps", fue objeto de especial atención por los ingleses, por lo que decididos a que no llegara a destino, llegaron a planear un asalto al puerto para llevárselo o hundirlo, aunque la intervención del embajador inglés en Madrid, Sir Samuel Hoare, impidió que se llevara a cabo. Sin embargo, se encomendó especialmente a Croker la constante vigilancia de dicho barco, que al parecer no fue demasiado efectiva; la noche del 19 de marzo de 1942, el barco alemán picó sus amarras y se escapó sin problemas con destino a Túnez. Esta evasión sentó muy mal en el mando aliado, y especialmente al embajador inglés (Álvarez Laita y Domínguez LLosá, 2017).

Por este fracaso, se decidió el relevo de Richard Croker, designándose a otro hispanoparlante llamado Sylvanus Rupert Gore Edwards, diplomático inglés con experiencia en Iberoamérica y casado Zulema Barlet Gore-Edwards de origen argentino. No conocemos con exactitud la fecha de su llegada a Melilla, pero debió ser en torno a los meses de septiembre y octubre de 1942. Pero si complicada fue la representación de Croker en Melilla, aún lo fue más la de Gore Edwards. [6]

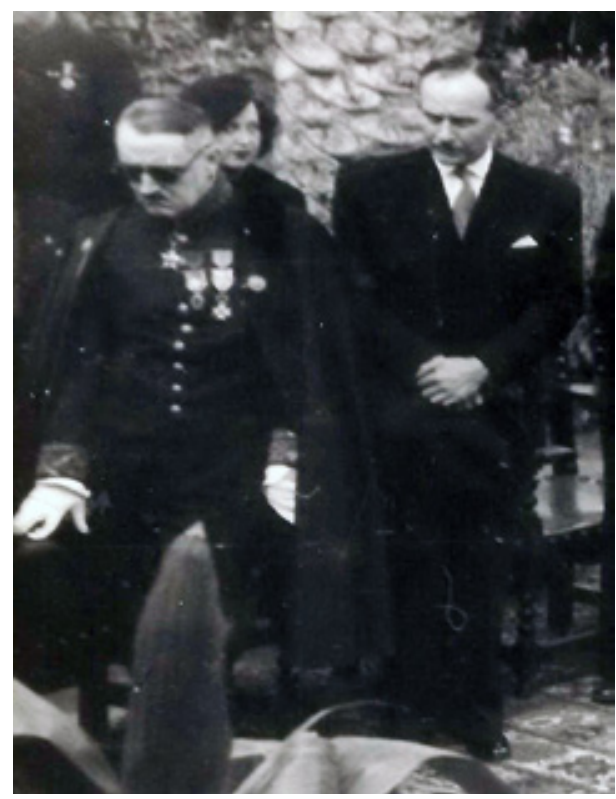

Fig. 6 Sylvanus R. Gore Edwars (derecha) junto al vicecónsul de Francia (Vichy) Henry Ribes (de uniforme) en un acto en 1943.

\footnotetext{
$11 \quad$ AGA. 81/4190. Expediente Personal de Richard Croker.

12 Archivo Intermedio Militar de Melilla (AIMM), $2^{a}$ Sección bis del EM del Ejército de Marruecos, nota informativa $n^{\circ}$ 58, de 12/02/1942 de la Oficina destacada $n^{\circ} 1$ (Melilla).

13 NA, Code 41 file 1223. FO 371/31268B, Incident at Melilla harbour regarding Dutch ship 'Lola' alias 'Faedjoer'.
} 
Descrito como un auténtico ejemplo de Lord inglés por sus modales y trato personal, en el aspecto profesional fue aún más duro que su antecesor. En el expediente que se conserva en el $\mathrm{AGA}^{14}$, son constantes los escritos en los que se describen enfrentamientos con autoridades y comerciantes. Quizás a este ambiente de hostilidad contribuyó el nombramiento del General Yagüe, de señaladas simpatías pro nazis, como máxima autoridad militar de la Zona Oriental del Protectorado. Su actitud hacia los intereses aliados y especialmente su trato ante el vicecónsul inglés, hizo que Gore Edwards describiera Melilla como "territorio enemigo" (Rhor, 2010, p. 176) ${ }^{15}$.

En el expediente mencionado se recogen varios incidentes, descritos tales como: "Se dedica a amenazar con la inclusión en listas negras a españoles e indígenas que pretenden comerciar con personas residentes en Melilla incluidas en las mismas". Otros incidentes fueron también grotescos, como cuando en marzo de 1943 hizo retrasar la salida del buque correo "Antonio Lázaro" por negarse a dar el correspondiente navicert al personal de una compañía de ópera italiana, a pesar de que todos sus integrantes fuesen españoles. También se dedicó a entorpecer el embarque de conocidos agentes alemanes, tales fueron los casos de Emilio Lacalle Luján en abril de 1943; o al conocido comerciante y representante de productos alemanes, Francisco Parres Puig, en octubre de 1943, que solo pudo embarcar finalmente tras la intervención del Comandante de Marina.

Hay que destacar asimismo el papel jugado por Gore Edwards en asuntos bélicos que se libraban a pocos kilómetros de Melilla. Como único cónsul aliado en la zona, se tenía que ocupar de los marinos y aviadores aliados que llegaban accidentalmente a la zona oriental del Protectorado. En noviembre de 1942, se hizo cargo de los paracaidistas norteamericanos que aterrizaron por error en Villa Sanjurjo y Tahuima, siendo constantes sus visitas a la Base Aérea de Tahuima, lugar donde fueron internados a la espera de su repatriación. Normalmente todos los militares aliados eran trasladados al poco tiempo a Gibraltar. Precisamente, el 2 de mayo de 1943, cuando traía a Melilla a aviadores aliados para que embarcaran, tuvo un incidente fronterizo al negarse a parar cuando cruzó la Aduana de Beni Enzar, lo

AGA. 81/10707. expediente personal de Sylvanus Rupert Gore-Edwards.

15 NA, FO 371/347779 C477, Carta de S.R. Gore Edwards a Gascoigne (Cónsul ingles en Tetuán). 
que motivó la correspondiente protesta de las autoridades españolas ante el Consulado General de Tetuán ${ }^{16}$. [7]

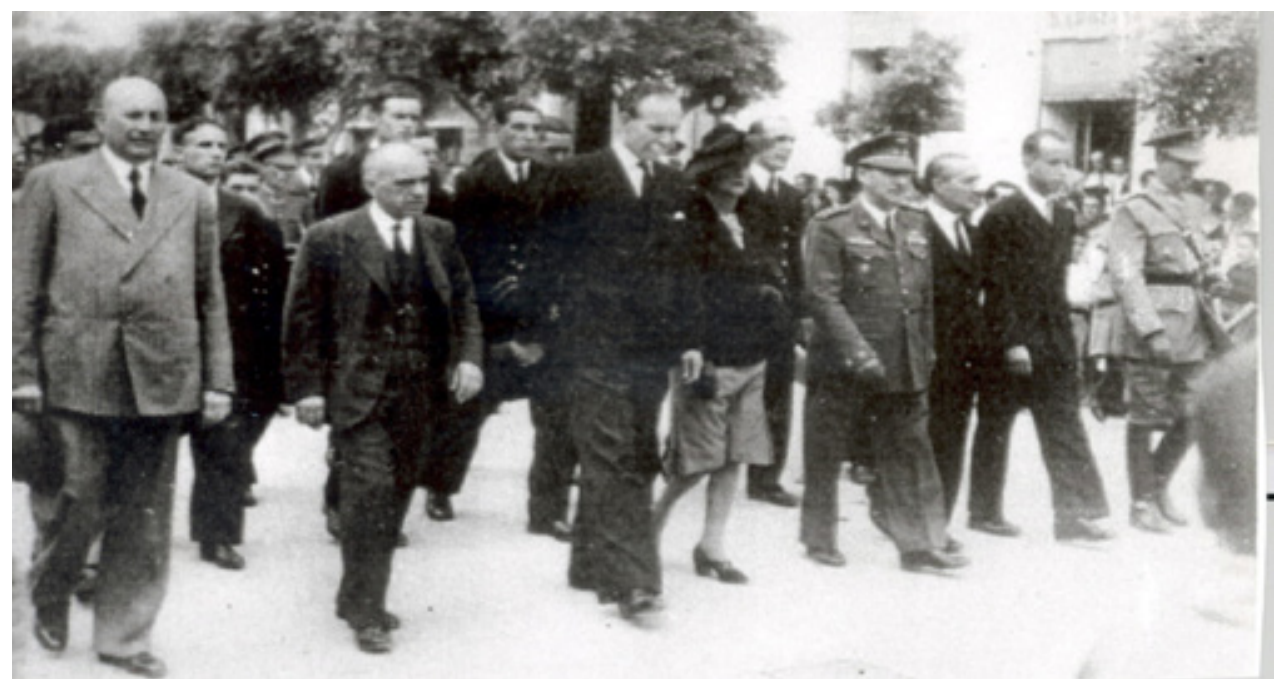

Fig.7 Cabecera del entierro de los aviadores ingleses que se estrellaron en el Cabo Tres Forcas. De izquierda a derecha, Emilio Piris Perea, canciller del viceconsulado inglés. Primer teniente de alcalde del Ayuntamiento de Melilla. Sylvanus R. Gore Edwards, vicecónsul inglés. Zulema Bartlet, esposa del vicecónsul inglés. Teniente coronel Guerrero, jefe de la Base Aérea de Tahuima.Vicecónsul de la Italia liberada. Representante oficioso de la Francia Libre. Comandante jefe de día de la Comandancia General. En la fila de atrás, oficiales de los buques mercantes ingleses presentes en el puerto de Melilla. Fotografía Manuel Cuenca Toro.

Otro incidente destacado ocurrió cuando el 16 de junio de 1944, un hidroavión Catalina PBY-5 del 202 escuadrón de la RAF (emplazado en Gibraltar), se estrelló al ser alcanzado por un rayo durante una tormenta en el Cabo Tres Forcas, pereciendo la vida sus nueve tripulantes. El entierro de estos aviadores británicos fue un acto multitudinario, presidido por Gore-Edwards. Durante el trayecto de la comitiva fúnebre, se produjeron algunos incidentes a la altura de la Plaza Comandante Benítez cuando miembros de la Falange, brazo en alto empezaron a cantar el Cara al Sol y a gritar vivas a Alemania y a Hitler, lo que motivó gestos de desagrado y quejas de la esposa del vicecónsul, Zulema Gore-Edwards ${ }^{17}$. [8]

\footnotetext{
16 AGA. 81/10707, Expediente personal de Sylvanus Rupert Gore-Edwards.

17 AGA (15) 13.1 81/2070 Expediente de Zulema Barlet Gore-Edwards.
} 


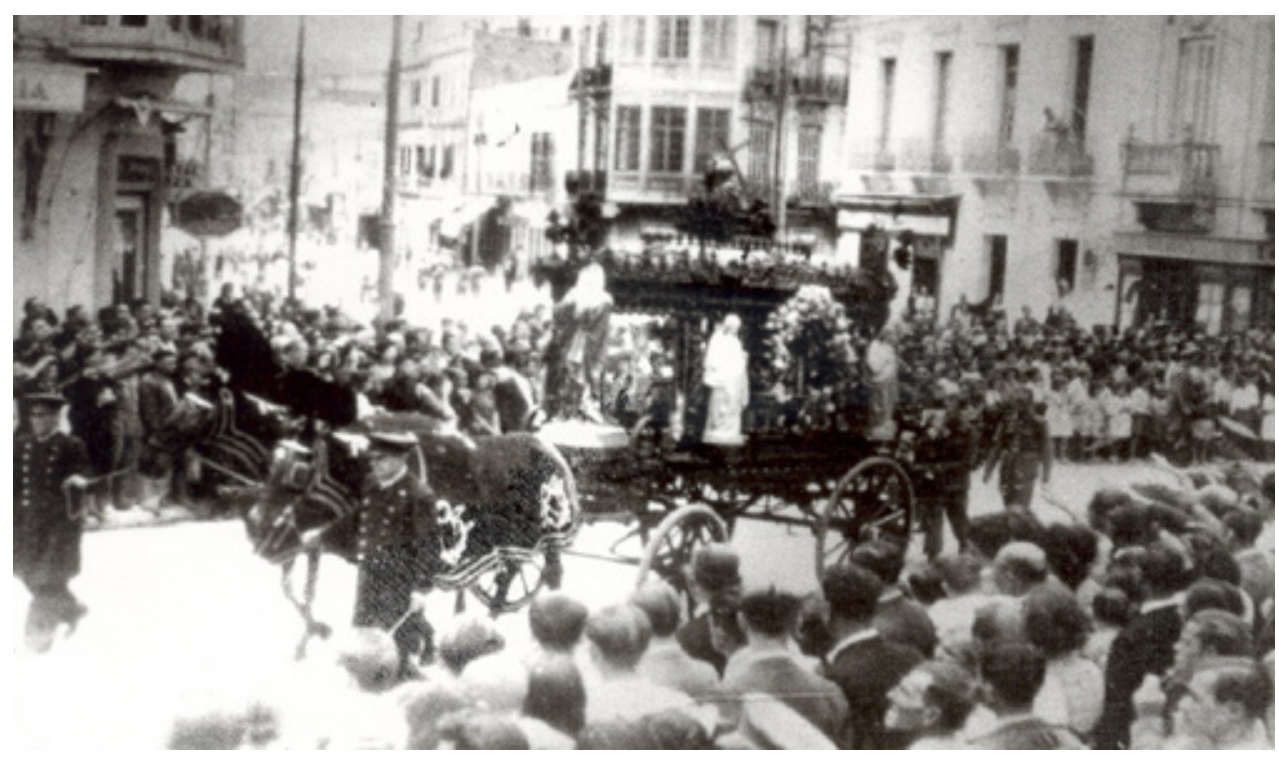

Fig. 8. Incidente ocurrido durante el entierro de los aviadores ingleses fallecidos en accidente en el Cabo Tres Forcas. A la izquierda y en la parte inferior derecha se aprecia el grupo de falangistas que entonaron el Cara al Sol y profirieron vivas a Franco, Hitler y Mussolinni al paso del cortejo fúnebre. Fotografía Manuel Cuenca Toro.

En abril de 1945, casi finalizada la guerra en Europa, y por motivos de salud, Sylvanus Rupert Gore-Edwards fue relevado. Esta vez por un australiano, Robert Keys Irving. Días después, el 4 de mayo, Gore-Edwards, y a bordo del vapor inglés Krapool, abandonó Melilla junto a su esposa con destino a Gibraltar. No realizaría mal su trabajo en Melilla ante los ojos de sus superiores, ya que posteriormente sería recompensado con el cargo de Embajador de Inglaterra en Argentina.

Aparte de los cónsules, el Viceconsulado inglés tenía otro personal destinado en el mismo. El secretario del mismo, era un gibraltareño afincado desde mucho tiempo atrás en Melilla llamado Emilio Piri Perea. Descrito como una persona afable, estaba totalmente integrado en la sociedad melillense, ostentando incluso algún cargo en una de las Cofradías de la Semana Santa melillense. Fue el encargado del Intelligence Service y de mantener al día la "lista negra" del consulado. En la década de los años cincuenta, fue nombrado vicecónsul honorario de Gran Bretaña en Melilla, siendo el último del que tenemos constancia en la ciudad.

Asimismo, consta la presencia como empleado del viceconsula- 
do de Marcel Louis Guirard. Esta persona, era en realidad capitán de la $\mathrm{RAF}^{18}$ y también agente del IS, realizando continuos viajes por la zona. En abril de 1943, abandonó Melilla para reincorporarse al servicio activo.

Del transporte de la valija diplomática se encargaba habitualmente otro gibraltareño, Joseph Charles Imossi, destinado primero en el viceconsulado de Tetuán, fue nombrado con posterioridad vicecónsul en Ceuta. Visitaba con mucha frecuencia Melilla.

\section{Consulado norteamericano.}

No hay constancia de la presencia en Melilla de ninguna representación oficial norteamericana en Melilla con anterioridad a la II Guerra Mundial.

Sin embargo, la ciudad si había sido visitada por distintos norteamericanos en viajes claramente informativos, como el realizado por el coronel William Chamberlain Bentley, pionero de aviación, y uno de los diseñadores del famoso B-17 Fortaleza Volante. Estuvo en Melilla, cuando era agregado aéreo (Bentley Jr., 2010) ${ }^{19}$ en el Consulado de Tánger del 23 al 25 de octubre de 1941, visitando a las autoridades civiles y militares, y participando también, en algunas de esas "juergas" a las que era tan aficionado el vicecónsul inglés Richard Croker ${ }^{20}$. El 8 de noviembre de 1942, Bentley era el jefe del asalto aerotransportado de Orán, dentro de la Operación Torch. Los aviones partieron desde Inglaterra y tras atravesar la Península Ibérica, debían dirigirse a los alrededores de Orán donde debían saltar los paracaidistas de la 82 División. El coronel Bentley, conocedor de la zona, había indicado a los navegantes que, en caso de separarse la formación, deberían buscar el Cabo Tres Forcas para usarlo como referencia. El mismo Bentley fue el oficial de mayor graduación en el asalto aerotransportado, siendo capturado por los franceses al aterrizar en la zona oriental de la sebkha de Orán, y posteriormente puesto

\footnotetext{
18 Royal Air Force, Real Fuerza Aérea británica.

19 Existe una biografía del Coronel Bentley titulada The touch of greatness donde se narra el viaje del coronel por el Protectorado español y Melilla en esas fechas, en el que iba acompañado por su esposa Bárbara.

20 Nota informativa $n^{\circ}$ 681, de 27/10/1941 de la Oficina destacada $n^{\circ} 1$ (Melilla), de la $2^{a}$ Sección bis del EM del Ejército de Marruecos. Archivo Intermedio Militar de Melilla. Atkinson, Rick. Un ejército al amanecer, Ed. Crítica, Barcelona 2002. Págs. 105 - 106.
} 
en libertad cuando acabó la resistencia francesa. ${ }^{21}$ [9]

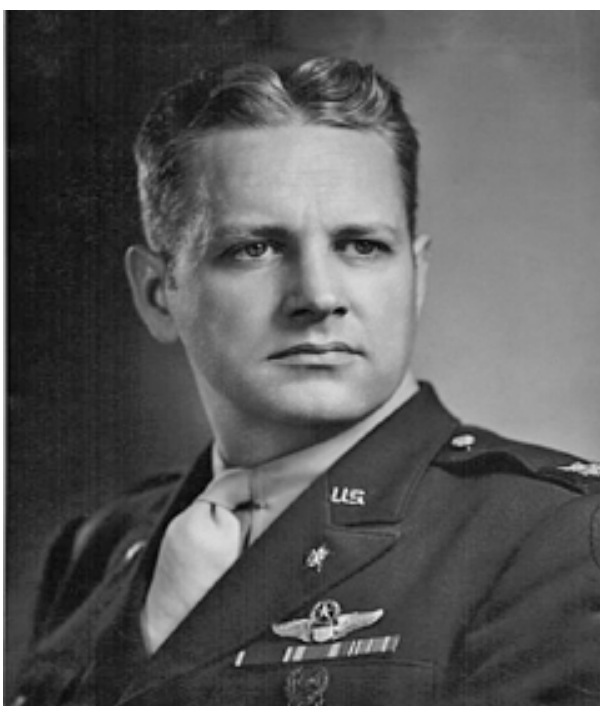

Fig.9 El coronel William Bentley, de la fuerza aérea de los EEUU.Visitó Melilla en 1941 con fines informativos cuando era agregado en el consulado americano en Tánger. Posteriormente, el 8 de noviembre de 1942 mandó el asalto aéreo durante la operación Torch.

No es hasta junio de 1943 cuando desde la Embajada de los EEUU se plantea la necesidad de instalar un consulado en Melilla.

El 5 de junio de ese año, J. Rivers Childs, Encargado de negocios norteamericano en Tánger, escribe una carta ${ }^{22}$ al Alto Comisario, General Orgaz, recordándole que en una conversación que ambos mantuvieron en Uxda, ya le había comunicado la intención del gobierno americano abrir un consulado en Melilla. Mientras el gobierno español autorizaba su apertura, Childs proponía el nombramiento de un funcionario afecto al consulado de Tánger, al que se le autorizaría a "residir temporal y oficiosamente en Melilla a fin de atender a la protección de los intereses americanos en sus inmediatas vecindades".

$\mathrm{Y}$ esos intereses, lo concreta a continuación:

Como ya conoce V.E., un cierto número de aviones se vieron forzados a aterrizar en los alrededores de Melilla, lo que nos obligó bien a enviar desde Tánger, con gran molestia a un representante ${ }^{23}$ al objeto de atender a las tripulaciones de aquellos, bien a delegar con tales propósitos al Vicecónsul británico en Melilla. Otras cuestiones pueden surgir de vez en cuando y sería de desear las pudiera tratar sobre el terreno un representante americano ${ }^{24}$. [10]

En esa misma carta se proponía ocupar este cargo al Secretario

21 Atkinson, Rick. Un ejército al amanecer, Ed. Crítica, Barcelona 2002. Págs. 105 - 106.

$22 \quad A G A$. Sección África. 81/10708. Expediente personal de Fayette F. Flexer.

23 Consta la llegada a Melilla el 3 de diciembre de 1942 del funcionario del Consulado en

Tánger Leonard Cecil Force, con "ropa, víveres, tabaco y otros enseres para los internados en Tahuima". Asimismo, el 2 de enero de 1943 son Douglas Flood y fames Merimon Dionell, secretario y oficial, respectivamente, del Consulado de Tánger, los que visitan a los paracaidistas y aviadores de Tahuima. 


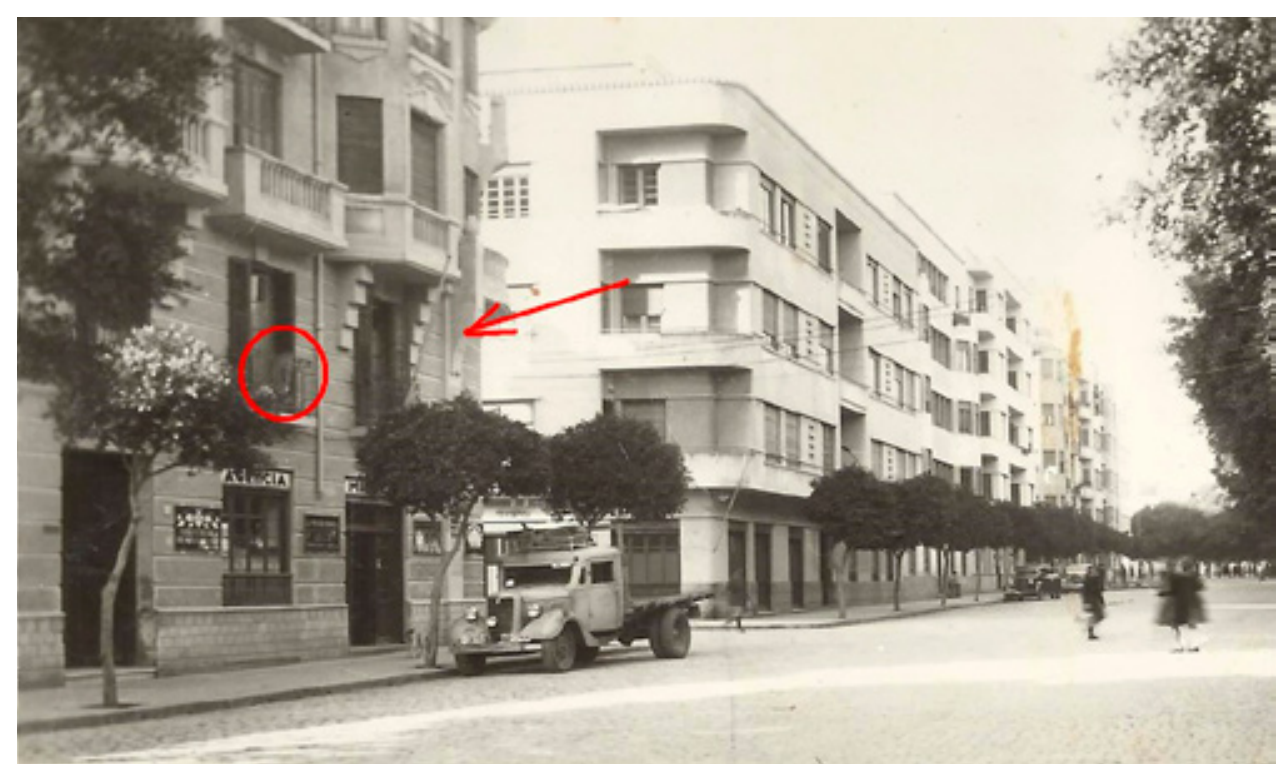

Fig. 10. La calle Teniente Coronel Segui en 1944. Señalada con el círculo, la placa del consulado norteamericano en Melilla. En el balcón de al lado, el mástil de la bandera.

de Embajada de $1^{\text {a }}$ clase Fayette J. Flexer, al cual fue autorizado a ocupar el puesto el día 10 de junio. Esta autorización provisional y oficiosa, no se hizo real hasta el 24 de agosto de 1943, cuando el Gobierno español otorga el preceptivo "Exequátur ${ }^{25}$ ", para que ocupase su puesto de Cónsul en Melilla. El Consulado de EE. UU se instaló finalmente en la calle Teniente Coronel Seguí, número 3.

El primer cónsul, Fayette J. Flexer natural de Joilet (Illinois) permaneció en su cargo hasta el 24 de mayo de 1944, cuando fue susti-

24 A lo largo de la II Guerra Mundial aterrizaron o cayeron en las cercanías de Melilla los siguientes aparatos aliados:

- 8/11/1942.- 1 C-47 en el aeródromo de Herrainz (Alhucemas) y otro en Monte Arruit.

- 12/11/1942.- 3 Spitfire Mk.V en el aeródromo de Tahuima.

- 01/1943. 1 B-24 en Tistutin

- 19/04/1943.- 1 P-38 Ligthnigth en la playa de la Bocana

- 25/04/1943. 1 Bristol Blenheim inglés cae al $N$ de las Chafarinas. Tripulantes rescatados por un pesquero melillense.

- 16/06/1944.- 1 PBY5 "Catalina” inglés se estrella en Cabo Tres Forcas. Mueren los

9 tripulantes.

- 04/08/1944. - 1 B-25 D en el aeródromo de Tahuima.

25 Exequátur consular: autorización que otorga un fefe de Estado a diplomáticos extranjeros para que en su territorio puedan ejercer funciones propias de sus cargos 
tuido por Vernon I. Fluharty, un experimentado diplomático que había realizado su carrera en varios países iberoamericanos.

La labor de los cónsules norteamericanos fue mucho más discreta que la de sus homólogos británicos, acudiendo solo en contadas ocasiones a recepciones oficiales, quizás debido a que al tratarse de personal diplomático de carrera ocasionaban un pequeño problema de protocolo al tener preeminencia sobre el resto del personal acreditado que se trataban de cargos honoríficos, algo que según los informes no era del agrado, por ejemplo, del representante francés, al que por antigüedad le correspondía encabezar las comitivas y besamanos hasta la llegada de los norteamericanos. No constan informes de incidentes con las autoridades, aunque también mantenían "listas negras" en las que figuraban numerosos melillenses. Estas listas negras, denominadas oficialmente "Proclaimed list of certain blocked nationals" 26 " eran constantemente revisadas, añadiendo o borrando a personas y empresas si se relacionaban o no con los países del Eje.

Otra de las misiones encomendadas al Consulado era el reparto de propaganda, distribuyéndose distintas revistas en español, profusamente ilustradas, como "Barras y Estrellas"27.

\section{II.- SERVICIOS SECRETOS ALIADOS EN MELILLA. DEL INTELLIGENCE SERVICE A LOS BANANA BOYS.}

\section{E1 "Intelligence Service" (IS)}

Como todo servicio de espionaje, la principal labor del IS en Melilla fue la obtención de información que pudiera ser útil para la causa aliada. Los datos obtenidos no se limitaban sólo a cuestiones militares, sino que abarcaban otras áreas como la economía, la cultura, la sociedad, etc. En los National Archives británicos de Gardens Kew, se conservan varios expedientes, elaborados entre los años

\footnotetext{
26 En Internet se pueden ver, digitalizadas, varias de estas listas negras norteamericanas. Por ejemplo, en la biblioteca on line de la Universidad de Michigan: http://catalog.hathitrust.org/

27 Según el testimonio de personas que vivieron en esos años, muchos niños de Melilla probaron por primera vez el chicle cuando acudian al Consulado norteamericano y los funcionarios se los regalaban. También coinciden en que las revistas norteamericanas de propaganda eran mejores que las inglesas y alemanas.
} 
1939 y 1946, en los que podemos observar el interés por la zona de Melilla y sus alrededores, en cuestiones que iban desde el comercio en el Protectorado (la importación de té inglés, la exportación de esparto, o problemas como la falta de moneda fraccionaria), como a temas estrictamente militares (movimientos de tropas, situación de aeródromos y su aprovisionamiento de combustible, bombas y munición $)^{28}$, pasando por análisis de la actitud de la población nativa ante la guerra ${ }^{29}$, y por supuesto, el seguimiento de las actividades de súbditos y agentes alemanes e italianos en la zona ${ }^{30}$.

Toda esta información se recibía a través de una extensa red de confidentes, informadores y agentes. En uno de los documentos que, temporalmente, fueron desclasificados en el Archivo Intermedio Militar de Melilla, se citan al menos 54 personas sospechosas de trabajar para el IS en Melilla. Destaca la presencia de numerosos hebreos, muchos de ellos protegidos del consulado británico o directamente súbditos de Su Graciosa Majestad. La dirección de esta red estaba a cargo del secretario del viceconsulado, el mencionado gibraltareño Emilio Piri Perea, y del consignatario de buques griego Giorgio S. Amanatides.

\section{Los Servicios Secretos norteamericanos (OSS)}

La actuación de los servicios de inteligencia británicos contrasta con la realizada por sus homólogos norteamericanos. Si los primeros se caracterizaron por limitarse a labores informativas, con la máxima discreción, los segundos, además, se dedicaron a la acción directa contra los intereses del Eje. Aunque con anterioridad ya habían visitado la ciudad algunos agentes norteamericanos, no es hasta los desembarcos aliados de noviembre de 1942 cuando más se hacen notar en la zona.

Por Melilla y sus alrededores pasaron algunos de los elementos más destacados de la OSS, como el mismo General Donovan, jefe del servicio, que estuvo en Uxda. Pero sobre el terreno, y en las calles de

28 NA, FO 371/24062 Troop movements in Spanish Morocco; CAB 84/15/53 Possible Spanish Action against French Morocco:1940 June 26.

NA. FO 371/26935. Attitude of Moors in Spanish Zone of Morocco. 1941.

30 NA, FO 371/31200 German activities in Spanish Morocco. 1942; KV 3/241 German espionage in Gibraltar, including Tangier and Morocco. 1940 fan 01 - 1944 Dec 31. 
Melilla, nos encontramos, entre otros, a dos famosos agentes secretos: Donald D. Downes ${ }^{31}$ y Carleton S. Coon.

El segundo de ellos era un viejo conocedor de la zona. Por su condición de antropólogo ya había recorrido la zona del Rif durante los años 20 y publicado varios estudios sobre los rifeños. Fue uno de los más activos agentes en los días previos a los desembarcos aliados en el Norte de África, y como anécdota, el inventor de los "excrementos explosivos ${ }^{32}$ ", que distribuyó por toda la zona de retirada de las fuerzas del Eje.

Con respecto a Downes, es considerado como el agente más completo que tuvo nunca la OSS, y su presencia en la zona está documentada desde la Guerra Civil ${ }^{33}$. Uno de sus mayores logros como agente del OSS, fue la infiltración en la Embajada de España en Washington, obteniendo los libros de códigos secretos y rompiendo así las comunicaciones entre la embajada y Madrid (Moreno, 2013).

En el año 1942, antes incluso de la Operación Torch, Downes ya había empezado a trabajar en la creación de equipos clandestinos para operar en España en el caso de que entrara en guerra a favor del Eje. Además de ello, se reunió con destacadas personalidades republicanas en el exilio, como Julio Álvarez del Bayo o José Aguirre (Pizarroso Quintero, 2009, p. 112). Gracias a ello pudo reunir y reclutar a una veintena de agentes españoles y norteamericanos, algunos de estos

31 Para seguir las actividades de Donald Downes y la Operación Banana en la zona, hemos seguido las siguientes fuentes: WINKS, Robin: Cloak and Gown. Scholars in America's Secret War. Ed. Collins Harvill, Londres, 1987. SMITH, R. Harris: OSS. The Secret History of America's First Central Intelligence Agency., University of California Press. Berkeley, 1972. SMITH, Bradley F.: The Shadows Warriors. OSS and the Origins of the CIA. Basic Books. Nueva York, 1983. PIZARROSO QUINTERO, Alejandro: Diplomáticos, Propagandistas y Espias. Estados Unidos y España en la Segunda Guerra Mundial. Información y Propaganda. CSIC. Madrid, 2009. SALINAS, Alfred. Les Américains en Algerie. 1942-1945. L'Harmattan. Paris, 2013. DOWNES, Donald.: The Scarlett Thread. Panther Books, Londres, 1970. AZUAGA RICO, fosé.: La guerrilla antifranquista en Nerja. Izquierda Unida-Los Verdes- Convocatoria por Andalucía. Nerja, 1996.

32 En sus visitas previas al norte de África, Carleton S. Coon habia advertido la abundancia de excrementos procedentes de animales en las carreteras y caminos. Ante ello, se le ocurrió darles esa forma y color a trozos de explosivo plástico que, con su correspondiente detonador de presión, eran colocados por agentes infiltrados en la retaguardia enemiga para sembrar el caos en las comunicaciones. Para más información: ARSUAGA, fuan Luis: Boñigas de camello. Diario El Pais, edición del 7 de noviembre de 2001.

33 AGA.81/10795, Expediente de DonaldV.L. Downes. 
fueron integrantes de la Brigada Lincoln durante la Guerra Civil. Estos agentes bajo las órdenes del agente Downes fueron conocidos como los "Donald Ducks" (Patos Donald en español, en clara alusión al nombre de su jefe) (Winks, 1987, p. 188).

Una vez que hubieron desembarcado los aliados en el Marruecos francés, Downes y su equipo se pusieron a las órdenes del G-2 (Intelligence Division) del V Ejército del general Clark, establecido en Uxda. A unos $70 \mathrm{Km}$. de esta localidad instaló su base Downes, encubierta como una estación meteorológica para poder infiltrar agentes en el Marruecos español, y posteriormente, en el mismo territorio peninsular. Pero su primera misión que se les encomendó fue la de destruir las redes de espionaje españolas, al servicio de Alemania y dirigidas desde Melilla por el Vicecónsul alemán Kraemer.

Primeramente, Downes logró infiltrar a un agente en la escuela de saboteadores que bajo dirección alemana funcionaba en Melilla. La infiltración fue tan profunda y eficaz, que según cuenta el propio Downes, "teníamos conocimiento de la misión de los equipos de saboteadores, antes de que salieran de la ciudad" (Downes, 1970). Esto eliminó uno de los mayores temores que tenían en el cuartel general de Clark, que desde el Protectorado español se realizaran acciones de sabotaje contra las líneas de suministros que iban desde los puertos marroquíes del Atlántico hasta el frente tunecino ${ }^{34}$.

Otra de las misiones encomendadas a Downes y su grupo, fue la neutralización de los agentes alemanes que operaban en Melilla. Para ello diseñaron un plan para atraer a algunos de ellos, a la zona francesa, donde serían capturados por los servicios aliados. Ante la prohibición expresa del general Clark de que actuaran en territorio bajo dominio español, el plan preveía atraer a los alemanes a una isla en medio del Muluya donde montarían la emboscada. Aunque se inició la operación, los alemanes no se presentaron en el lugar.

Actuando al margen de la Embajada estadounidense, pero con el beneplácito del general Donovan, Downes empezó a organizar una infiltración a gran escala en España. Esa acción recibió en su primera

\footnotetext{
$34 \quad$ Pese a todo hubo algunos intentos de sabotaje sin mayores consecuencias. Los servicios secretos aliados consiguieron neutralizar a todos los saboteadores que cruzaban la frontera pese a que en muchos casos contaban con la connivencia de las autoridades francesas (Salinas, 2013, p. 231 y siguientes).
} 
fase el nombre de "Operación Banana" 35 , y por ello, sus componentes eran conocidos, como los "Banana Boys"36.

En junio de 1943, se lanzó la primera fase enviando a Málaga, en una pequeña barca de pesca, al primer equipo. Poco después, mandaba otros dos equipos a Cádiz y Melilla.

El equipo enviado a Melilla, que tenía el nombre en clave de "apricott", constaba de dos agentes: Garrido, como informador, y Salvador Rodríguez Santana, como radiotelegrafista. En agosto de 1943 $(\text { Navarro, 1999 })^{37}$, después de haber recibido entrenamiento en Argel, los dos agentes españoles, junto a tres instructores militares norteamericanos marcharon a Uxda para esperar el momento oportuno de pasar la frontera.

Fue el 17 de septiembre, cuando en compañía de varios marroquíes, y camuflados como ellos, atravesaron la frontera. Tras pasar toda la noche caminando, llegaron a una cabila cercana a la localidad de Segangan, donde dejaron la radio y granadas de mano. Tras un corte descanso, al día siguiente por la noche, llegaron a Melilla donde fueron recibidos por el miembro del Partido Comunista local (PCE), Antonio Herrera.

A partir de ese momento, los dos agentes empezaron a vivir con la máxima discreción, intentado pasar inadvertidos en la militarizada ciudad. Cambian constantemente de alojamiento. Herrera les proporciona dos casas, una en la calle Horcas Coloradas $n^{\circ}$ 13, y la otra en calle Zoco Reina Regente. Pero ninguna les era útil por la sencilla razón de que carecían de acometida eléctrica.

El 7 de diciembre, Garrido regresa a Argel al haberse quedado sin fondos, siendo sustituido el día 24 por otro informador de nombre Julio. Éste se alojaría en el piso de la calle de Horno $\mathrm{n}^{\circ}$ 4, en Melilla la Vieja, propiedad de dos hermanos miembros del PCE: Juan y Agustín Coca, pero alquilada a nombre de José López Iglesias, Secretario General del

35 La Operación Banana I implicaba la infiltración de agentes en Melilla, Ceuta y Málaga. Las fases sucesivas también llevaban nombres de frutas: "Orange" (Cartagena), "Grapefruit" (Cádiz), "Apple" (Algeciras), "Cherry" (Barcelona) y "Lemon" (Madrid).

36 Para más información: LEAL, Favier. Operación Banana: Cuando la CIA y el Partido Comunista español espiaban juntos. En http://khronos. es/operacion-banana-cia-pcel

37 Para seguir las vicisitudes de los agentes al servicio de la OSS en Melilla ver el artículo fusto Navarro. También el reciente documental "Espías en la arena. Objetivo España" en http://www.rtve.es/alacarta/videos/otros-documentales/espias-arena-objetivo-esp/4428634/ 
partido en Melilla. Mientras tanto, la radio ya había sido transportada por otro miembro del PCE, Rafael Requena, mecánico de aviación y destinado en la cercana base de hidroaviones del Atalayón. También realizaría misiones de información y apoyo un cabo de Regulares, llamado Francisco Muriel, que se desvincularía de la organización a finales de 1943.

En la noche del 23 de febrero de 1944, Salvador Rodríguez fue a la casa de la calle del Horno, donde residía Julio. Se quedaría a dormir allí, al temer quedarse dormido y no poder hacer la transmisión prevista para las siete y media de la mañana. Ambos ignoraban que Garrido, el primer compañero de Salvador, ya había sido apresado en Málaga y los había delatado y que en la tarde el día anterior, se habían presentado ante el Comisario Jefe de Melilla, José Fresneda Cazorla, dos miembros de la Brigada Político Social de Madrid, los agentes Ramón González Bachiller y Bernardo Bachiller García que traían órdenes de la Dirección General de Seguridad "para llevar a efecto la detención de varios individuos, como así mismo el rescate u ocupación de una radio, armas y propaganda relacionada con la Unión Sovietica"38. Junto a la orden, presentan una nota con la delación que los había traído a la ciudad:

"Emilio; feo (cara de orangután); dientes salientes; representa unos treinta y cinco años; moreno; Usa algunas veces gafas ahumadas graduadas; vino la primera vez a Melilla yendo a parar a la calle de Vistahermosa número uno, casa de Luis Belinchón, que es pescador; este pasó sobre el mes de noviembre; de esta casa se lo llevó una tal Pepe a otra casa de al lado del Cementerio; la radio se la llevo el tal Pepe que vive en la calle del Horno número cuatro y allí vive con su mujer y su hija; es mecánico, de estatura regular; también vive en dicha casa un tal Juanito, delgado, alto, amigo de ambos es un tal Rafael, y un tal Muriel, de oficio impresor, alto, delgado, de unos veintisiete a veintiocho años; todos estos son presentados por Antonio Herrero; tienen una pistola americana del calibre cuarenta y cinco y otra italiana del mismo calibre corto, esta última la lleva un tal Julio, poseyendo material, datos de Pedro Garrido el mismo Juan Guirado.

Inmediatamente se ponen en marcha las pesquisas policiales, empezando por comisionar al inspector jefe de la Brigada de Información de Melilla, Julián Torralba Troncoso, "para que se persone en el Excmo.

38 Trascripción literal del expediente que sobre la muerte del inspector Fulián Torralba Navarro, se custodiaba en la Comisaría de Policía de Melilla hasta su traslado al Archivo de la Policía de Madrid. El autor pudo consultarlo en 2003. 
Ayuntamiento y recabe permiso a fin de poder ver y revisar los padrones de vecinos y calles de esta Ciudad para averiguar los domicilios de los sujetos expresados", diligencia realizada ya el día 23 al mediodía.

Con estos datos se monta el operativo que debía realizar las detenciones, compuesto, además de los dos inspectores llegados de Madrid, por el citado inspector Julián Torralba y los agentes Juan Lladó Lara, Rafael López Rosales, Mariano Díaz González, José mandado Romero, José Luis Arjona Castillo y Manuel Pinto Fernández.

A la una de la madrugada, cercan la casa, en la que entran los tres inspectores que comienzan a interrogar a Agustín Coca. Ante la poca colaboración de este, que repetía una y otra vez que allí solo estaban él, su mujer y su hijo, y una vez se le mostró una foto del llamado "Pepe" (en realidad José López Iglesias), manifestó que lo conocía, pero que no vivía allí, sino en la calle Duque de la Torre. Los tres inspectores registran la casa hasta que encontraron una puerta cerrada con llave. Revientan la puerta, y en la penumbra observan a dos personas en una cama, a los que se les da el alto. Uno levanta los brazos, pero el otro manipula algo bajo las mantas. De pronto, y antes de que Torralba, el más cercano a los sospechosos hiciera uso de su arma, se apaga la luz y en la oscuridad se escucha un disparo que alcanza al inspector. Los otros dos policías se repliegan, circunstancia que aprovechan los agentes del OSS para intentar huir por un patio. Pero a Salvador Rodríguez lo retiene un vecino hasta la llegada de la policía. El otro agente, Julio, intenta entrar en una casa de la calle San Miguel pistola en mano, pero es abatido por tres disparos de fusil de uno de los policías que lo alcanza.

Mientras tanto, en otros lugares de Melilla se irían produciendo más detenciones. Son apresados los hermanos Coca, José López Iglesias, Francisco Muriel, Rafael Requena y Mohamedi Ben Hadi Dudu, dueño de la casa donde se ocultó la radio.

En la casa de la calle del Horno se realizó un registro y bajo un montón de periódicos aparece la radio (de fabricación americana), armas y documentación.

Gracias a dicha documentación se supo cual era la información que habían obtenido los agentes: noticias de prensa y otras de carácter general y al alcance de cualquiera. Juan Coca, sin embargo, aportó datos sobre movimientos de agentes alemanes y tropas, obtenidos cuando acompañaba a Kraemer, vicecónsul alemán, debido a que trabajaba para él. También obtuvo más información por las visitas de este a la Oficina de estadística del Estado Mayor (servicio secreto). Pero la única información 
verdaderamente interesante, fue una lista de agentes alemanes del archivo de la Policía.

Realizada las oportunas investigaciones, el 23 de marzo de 1944, en la Sala de Banderas del Regimiento Mixto de Infantería ${ }^{\circ}$ 90, se constituyó un tribunal militar, bajo la presidencia del Teniente Coronel Francisco Álvarez, para juzgar en Consejo de Guerra Sumarísimo la causa n ${ }^{\circ}$ 1113/1944, contra los acusados Agustín y Juan Coca, Francisco Muriel, José López Iglesias, Rafael Requena, Salvador Rodríguez Santana, Mohamedi Ben hadi Dudu y otros, por los delitos de rebelión y espionaje. Pese a que el fiscal, teniente auditor José Civeira de la Cruz, solicitó la pena de muerte para todos excepto para Agustín Coca y Mohamedi Ben hadi Dudu, el tribunal no lo admitió, imponiendo solo penas de cárcel, siendo la más alta la impuesta a Salvador Rodríguez Santana: 12 años por espionaje y 20 por rebelión, en total 32 años de condena.

Pasada esta primera sentencia a instancias superiores, el teniente general Jefe del Ejército de Marruecos no la aprobó, ordenándose que se celebrara un nuevo Consejo de Guerra el 10 de abril, presidio esta vez por el teniente Coronel Manuel Rodríguez. Y pese a que el acta es idéntica, las sentencias cambian sustancialmente: Pena de muerte para Salvador Rodríguez Navarro, José López Iglesias y Francisco Muriel Martín.

La sentencia fue ratificada por el Alto Comisario general Orgaz el 24 de abril, siendo ejecutados los condenados en Rostrogordo, a las siete de la mañana del 3 de agosto de 1944.

Pero este incidente tuvo más repercusiones. A los detenidos en Melilla hay que sumarles los que lo fueron en Ceuta y Málaga. En total, fueron once los componentes de los "Banana Boys" que murieron en la fracasada operación, lo que llevó al mismo Downes a rebautizarla como Operación Bloody Bananas.

También tuvo su trascendencia política y diplomática. La operación se había realizado sin conocimiento del Embajador norteamericano, el cual, al enterarse del fracaso, ordenó que se cancelara cualquier operación en marcha sin su permiso. Donovan, el jefe de la OSS, en un primer momento negó saber nada al respecto, aunque acabó reconociendo que la había planificado. El Gobierno español, que en esos momentos estaba siendo muy presionado por los aliados, recibió este fracaso como un balón de oxígeno, presentando una protesta diplomática contra ellos. El Embajador norteamericano, Carlton Hayes, se vio obligado a pedir disculpas por lo ocurrido, y a partir de ese momento, todas las operaciones que se realizaran en España deberían ser aprobadas por el Embajador. 


\section{Epílogo}

No constan, al momento y a la espera de que la desclasificación de archivos aporte nuevos datos, ningún otro movimiento de los servicios secretos aliados en Melilla una vez conseguida la neutralización, al final de la guerra de los servicios de inteligencia del Eje.

Con el fin de la contienda mundial, y ante la situación de aislamiento a la que fue sometida la España franquista, dejaron de tener sentido la presencia de los consulados en Melilla. El primero en cerrar fue el norteamericano en 1946. Unos años más duró en inglés, que siguió abierto hasta mediados de los años 50.

\section{Bibliografía}

ÁLVAREZ LAITA, Francisco Javier y DOMÍNGUEZ LLOSÁ, Santiago (2017). El extraño caso del Jaedjoer alias Lola, en Revista de Historia Naval, $n^{\circ} 136$, p. 81 a 102.

ARSUAGA, Juan Luis (2001), "Boñigas de camello". Diario El País, edición del 7 de noviembre de 2001.

AZUAGA RICO, José (1996). La guerrilla antifranquista en Nerja. Izquierda Unida-Los Verdes- Convocatoria por Andalucía. Nerja.

BENTLEY, Stewart W. Jr. (2010) The touch of greatness. Ed. Authorhouse, Bloomington, Indiana.

DOWNES, Donald (1970) The Scarlett Thread. Panther Books, Londres.

MORENO, Rafael (2013), "Espías en el sitio más extraño”. Diario El País, edición de 08/11/2013.

NAVARRO, Justo (1999), "Los espías de Ike en Melilla", diario El País, el domingo 21 de febrero de 1999.

PIZARROSO QUINTERO, Alejandro (2009), Diplomáticos, Propagandistas y Espías. Estados Unidos y España en la Segunda Guerra Mundial. Información y Propaganda. CSIC. Madrid.

ROHR, Isabelle (2010), La derecha española y los judíos. Universidad de Valencia. Valencia.

SALINAS, Alfred (2013), Les Américains en Algerie. 1942-1945. L'Harmattan. Paris.

SMITH, R. Harris (1972), OSS. The Secret History of America's First Central Intelligence Agency, University of California Press. Berkeley.

SMITH, Bradley F. (1983), The Shadows Warriors. OSS and the Origins of the CIA. Basic Books. Nueva York.

WINKS, Robin (1987), Cloak and Gown. Scholars in America's Secret War. Ed. Collins Harvill, Londres. 